\title{
Superatom Picture of Collective Nonclassical Light Emission and Dipole Blockade in Atom Arrays
}

\author{
L. A. Williamson, ${ }^{1}$ M. O. Borgh $\odot{ }^{2}{ }^{2}$ and J. Ruostekoski ${ }^{1}$ \\ ${ }^{1}$ Department of Physics, Lancaster University, Lancaster LA1 4YB, United Kingdom \\ ${ }^{2}$ Faculty of Science, University of East Anglia, Norwich NR4 7TJ, United Kingdom
}

(Received 8 May 2020; accepted 10 July 2020; published 13 August 2020)

\begin{abstract}
We show that two-time, second-order correlations of scattered photons from planar arrays and chains of atoms display nonclassical features that can be described by a superatom picture of the canonical singleatom $g_{2}(\tau)$ resonance fluorescence result. For the superatom, the single-atom linewidth is replaced by the linewidth of the underlying collective low light-intensity eigenmode. Strong light-induced dipole-dipole interactions lead to a correlated response, suppressed joint photon detection events, and dipole blockade that inhibits multiple excitations of the collective atomic state. For targeted subradiant modes, the nonclassical nature of emitted light can be dramatically enhanced even compared with that of a single atom.
\end{abstract}

DOI: 10.1103/PhysRevLett.125.073602

The first direct evidence for the quantum nature of light was observed in resonance fluorescence of an atom [1-5], defining a significant historical milestone in quantum optics. Such quantum correlations can be identified by measuring the second-order correlation function for the emitted field that represents a joint probability of two photon detection events appearing a time $\tau$ apart and can be defined as

$$
g_{2}(\tau) \equiv \lim _{t \rightarrow \infty} \frac{\langle: \hat{n}(t+\tau) \hat{n}(t):\rangle}{\langle\hat{n}(t)\rangle^{2}}
$$

where :: denotes normal ordering and $\hat{n}(t)$ is the number operator for detected photons. Classically, $g_{2}(0) \geq g_{2}(\tau)$; hence $g_{2}(0)<g_{2}(\tau)$ implies quantum correlations in the photon emission, and also defines antibunched photon emission [6,7].

Going beyond a single atom, in a noninteracting ensemble atoms will emit photons independently, leading to an adulteration of the single-atom photon antibunching that (neglecting interferences) scales as $1-N^{-1}$ with the atom number $N[3,8,9]$. Correlated excitations for atomic ensembles have been observed for highly excited Rydberg atoms in the microwave regime. The correlated response is generated by dipolar interactions that inhibit transitions into all but singly excited states, representing dipole blockade [10-16], with applications to scalable quantum logic gates.

In dense ensembles of cold atoms, also light-mediated interactions between the atoms can lead to drastic and unexpected phenomena [17-20] as multiple resonant scattering events give rise to a correlated response. Correlations can emerge even for the classical optical regime in the limit of low light intensity (LLI) of an incident laser [21,22], and the quest for observing the effects of strong light-mediated interactions is attracting considerable attention [23-34]. Regular arrays of atoms are particularly interesting for the exploration and manipulation of collective optical responses, as more recently studied also in the quantum regime [35-47]. Transmission-resonance narrowing due to collective subradiance in the classical limit in a planar optical lattice was already observed [48] and other related experiments are rapidly emerging [49].

Here we show that photon emission events from planar arrays and chains of atoms can still be described by the single-isolated-atom picture, representing a collective response of the entire atomic ensemble as one superatom. By resonantly targeting LLI collective excitation eigenmodes, we show that even at high light intensities the many-atom joint photon emission $g_{2}(\tau)$ displays the same functional form as the single-isolated-atom $g_{2}(\tau)$ of Eq. (1), but with the single atom linewidth replaced by the linewidth of the targeted LLI collective mode. We find that for sufficiently small lattice spacings strong light-induced interactions can increase antibunching by establishing correlations between the atoms that represent inhibited multiple excitations of the collective state of the atoms, or dipole blockade. Remarkably, for underlying LLI eigenmodes for which the resonance linewidth is much narrower than the one for an isolated atom (subradiance), the nonclassical nature of emitted light can be dramatically enhanced to much longer timescales even compared with those of a single atom.

We consider two-level atoms with the dipole matrix element $\mathbf{d}$, coupled by light-mediated interactions and subject to an incident laser field. The atom dynamics in the rotating-wave approximation follows from the manybody quantum master equation (QME) for the reduced density matrix [50-52] 


$$
\begin{aligned}
\frac{d \rho}{d t}= & -\frac{i}{\hbar} \sum_{j}\left[H_{j}, \rho\right]+i \sum_{j \ell(\ell \neq j)} \Delta_{j \ell}\left[\hat{\sigma}_{j}^{+} \hat{\sigma}_{\ell}^{-}, \rho\right] \\
& +\sum_{j \ell} \gamma_{j \ell}\left(2 \sigma_{j}^{-} \rho \sigma_{\ell}^{+}-\sigma_{\ell}^{+} \sigma_{j}^{-} \rho-\rho \sigma_{\ell}^{+} \sigma_{j}^{-}\right)
\end{aligned}
$$

with the atomic operators $\hat{\sigma}_{j}^{+}=\left(\hat{\sigma}_{j}^{-}\right)^{\dagger}=|e\rangle_{j j}\langle g|$, $\hat{\sigma}_{j}^{e e}=\hat{\sigma}_{j}^{+} \hat{\sigma}_{j}^{-}$, for ground $|g\rangle_{j}$ and excited $|e\rangle_{j}$ states of atom $j$ located at $\mathbf{r}_{j}$ and

$$
H_{j} \equiv-\hbar \delta \hat{\sigma}_{j}^{e e}-\mathbf{d} \cdot \mathcal{E}^{+}\left(\mathbf{r}_{j}\right) \hat{\sigma}_{j}^{+}-\mathbf{d}^{*} \cdot \mathcal{E}^{-}\left(\mathbf{r}_{j}\right) \hat{\sigma}_{j}^{-} .
$$

We take the positive-frequency component $\mathcal{E}^{+}(\mathbf{r})=$ $\frac{1}{2} \mathcal{E}_{0} e^{i \mathbf{k} \cdot \mathbf{r}} \hat{\mathbf{e}}$ of the laser field to be a monochromatic plane wave of frequency $\omega=k c=2 \pi c / \lambda$ and wave vector $\mathbf{k}$, detuned from the single-atom transition frequency $\omega_{0}$ by $\delta \equiv \omega-\omega_{0}$. The light and atomic field amplitudes are here defined as slowly varying with the rapid oscillations at the laser frequency factored out. The light-mediated interactions between the atoms have both coherent $\Delta_{j \ell}$ and dissipative $\gamma_{j \ell}$ contributions $\left[\gamma_{j j}=\gamma \equiv|\mathbf{d}|^{2} k^{3} /\left(6 \pi \hbar \epsilon_{0}\right)\right.$ is the single atom linewidth]. These are the real and imaginary parts, respectively, of $\mathbf{d}^{*} \cdot \mathbf{G}\left(\mathbf{r}_{j}-\mathbf{r}_{\ell}\right) \mathbf{d} / \hbar \epsilon_{0}$, with $\mathrm{G}(\mathbf{r})$ the dipole radiation kernel of a point dipole at the origin $[52,53]$.

In the limit of LLI the dynamics reduces to that of classical coupled dipoles $[67,68]$. In this regime we may describe [52] the optical response using LLI collective radiative excitation eigenmodes $u_{m}$ of $\mathcal{H}_{j \ell}=\Delta_{j \ell}+i \gamma_{j \ell}$ (with $\Delta_{j j} \equiv 0$ ), with the complex eigenvalues $\zeta_{m}+i v_{m}$ representing the collective linewidth $v_{m}$ and line shift $\zeta_{m}$ from the single-atom resonance. The linewidths can span many orders of magnitude, from extremely subradiant to superradiant $[29,69,70]$.

To calculate the rate of the detected photons for the second-order correlation function $g_{2}(\tau)$ of Eq. (1) we assume all the scattered photons are detected and integrate $\hat{n}(t)=\left(2 \epsilon_{0} c / \hbar \omega_{0}\right) \int_{S} d S \hat{\mathbf{E}}_{\mathrm{sc}}^{-}(\mathbf{r}, t) \cdot \hat{\mathbf{E}}_{\mathrm{sc}}^{+}(\mathbf{r}, t)$ over a closed surface enclosing the atoms to give $\hat{n}=2 \sum_{j \ell} \gamma_{j \ell} \sigma_{j}^{+} \sigma_{\ell}^{-}$ [52], where $\epsilon_{0} \hat{\mathbf{E}}_{\mathrm{sc}}^{+}(\mathbf{r}, t)=\sum_{j} \mathrm{G}\left(\mathbf{r}-\mathbf{r}_{j}\right) \mathbf{d} \hat{\sigma}_{j}^{-}(t)$ denotes the scattered electric field summed over all the atoms. For a single isolated atom, a closed expression for $g_{2}(\tau)$ can be derived analytically and is given by [1,54],

$$
g_{2}^{(\gamma, \kappa)}(\tau) \equiv 1-e^{-3 \gamma \tau / 2}\left(\cosh \kappa \gamma \tau+\frac{3}{2} \frac{\sinh \kappa \gamma \tau}{\kappa}\right),
$$

where $\quad \kappa \equiv \frac{1}{2} \sqrt{1-8 I_{\text {in }} / I_{s}}, \quad$ and $\quad I_{\text {in }} \equiv \epsilon_{0} c\left|\mathcal{E}_{0} \hat{\mathbf{e}} \cdot \hat{\mathbf{d}}\right|^{2} / 2$ and $I_{s} \equiv \hbar c k^{3} \gamma / 6 \pi$ are the incident light and saturation intensities, respectively. Since $g_{2}^{(\gamma, \kappa)}(0)=0$ and $\lim _{\tau \rightarrow \infty} g_{2}^{(\gamma, \kappa)}(\tau)=1$, a single isolated atom therefore shows photon antibunching, a manifestation of the fact that an atomic energy level can contain at most a single excitation.
For the many-body system, $g_{2}(\tau)$ [Eq. (1)] in general needs to be evaluated by first solving the QME (2) numerically. The existence of nonclassical effects for a many-atom ensemble is less obvious than in the singleatom case. This can be illustrated by a simple counting example of $N$ independently emitting, noninteracting atoms: Neglecting interferences then yields $g_{2}(\tau)=1+$ $N^{-1}\left[g_{2}^{(\gamma, \kappa)}(\tau)-1\right]$, indicating a rapidly reduced photon antibunching as a function of the atom number, as photons from independently emitting atoms wash out the correlations.

For the case of strong cooperative coupling of closely spaced atoms we have a strongly correlated quantum manybody system with long-range dipole-dipole interactions. While we have also numerically calculated $g_{2}$ for such situations, our key observation is that for several strongly correlated regimes of interest, Eq. (4) remarkably can still provide a qualitative description for emitted photon correlations that also exhibit nonclassical scattered light and inhibited multiple excitations (dipole blockade) even for increasing atom numbers. This is because atoms collectively respond as one giant superatom, where effectively the single-particle resonance linewidth is replaced by the resonance linewidth of the dominant underlying LLI collective excitation eigenmode.

The dominant eigenmode in a regular array is determined by the resonance frequency and phase-matching profile with the incident field. We find then that the many-body $g_{2}(\tau)$ obeys a functional form analogous to Eq. (4),

$$
g_{2}(\tau) \approx 1+b\left[g_{2}^{\left(v, \kappa^{\prime}\right)}(\tau)-1\right]
$$

where $v=v_{\ell}$ is the linewidth of the resonant LLI eigenmode $u_{\ell}$ (found by diagonalizing $\mathcal{H}_{j \ell}$ [52]) and $\kappa^{\prime} \equiv$ $\frac{1}{2} \sqrt{1-8 \mathcal{I} I_{\text {in }} / I_{s}^{\prime}}$, with $I_{s}^{\prime} \equiv \hbar c k^{3} v / 6 \pi$. The overlap of the drive field with $u_{\ell}, \mathcal{I}=\left|\sum_{j} e^{-i \mathbf{k} \cdot \mathbf{r}_{j}} u_{\ell}\left(\mathbf{r}_{j}\right)\right|^{2}$, represents the sum of the coupling strengths of light over all the atoms and can for uniform targeted modes with perfect phasematching be replaced by $N$, reflecting the collective $N$-enhancement of the response. There is an overall normalization in Eq. (5) by $b \approx 1-g_{2}(0)$ that accounts for nonclassical light emission at zero delay due to manybody correlations. When $b>N^{-1}$, these are enhanced compared to the noninteracting, noninterfering case.

In the numerics, we consider 2D square arrays of atoms in the $x y$ plane and 1D chains along the $x$ axis, with the incident light direction $\hat{\mathbf{k}}=\hat{\mathbf{z}}$, polarized along the atomic dipoles $\hat{\mathbf{d}}=\hat{\mathbf{x}}$. We solve the QME by directly integrating Eq. (2) or by unraveling the evolution into stochastic quantum trajectories of state vectors [52,55-57,71].

We demonstrate nonclassically scattered light from a strongly interacting $3 \times 3$ planar array of atoms in the twotime correlation function in Fig. 1, where the nonclassicality of the photon emission is strongly enhanced due to 

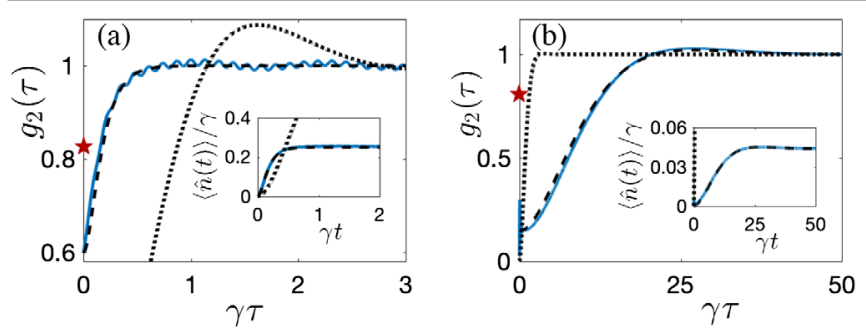

FIG. 1. Superatom picture and nonclassical light scattering for a $3 \times 3$ atom array (lattice spacing $a=0.1 \lambda$ ) with a drive field resonant with (a) the uniform superradiant $\left(v \approx 7.6 \gamma, N I_{\text {in }}=2 I_{s}\right)$ and (b) a subradiant ( $\left.v \approx 0.091 \gamma, N I_{\text {in }}=0.5 I_{s}\right)$ LLI collective eigenmode; $g_{2}(\tau)$ for the full quantum solution (blue solid line), superatom (black dashed line), and single isolated atom (black dotted line). The red star marks the noninteracting, interfering result of $g_{2}(0)$, showing that interactions substantially enhance photon antibunching. For the subradiant mode the nonclassical emission is enhanced compared with a single atom. Insets show the corresponding photon detection rates.

interactions. This corresponds to inhibited multiple excitations of the collective atomic state due to light-mediated dipole-dipole interactions, representing dipole blockade of optical transitions, analogous to collective suppression of microwave Rydberg excitations [11]. The drive, which is uniform across the plane, couples most strongly to the most superradiant LLI eigenmode with no phase variation across the atoms. We show that the superatom picture (SAP) [Eq. (5)] provides an excellent description of $g_{2}(\tau)$ for light resonant with this mode $(v \approx 7.6 \gamma, \mathcal{I} \approx 0.98 N)$ [Fig. 1(a)]. The antibunching delay time is much shorter than that of a single atom.

The incident light can also be tuned to target a subradiant eigenmode. Here we consider the eigenmode with the fourth broadest resonance, with $v \approx 0.091 \gamma$ and $\mathcal{I} \approx 0.015 N$. We find that the SAP again accurately describes the dynamics [Fig. 1(b)]. The mode is approximately $u_{\ell}\left(\mathbf{r}_{j}\right) \approx$ $1.4 \cos \left(\pi \hat{\mathbf{x}} \cdot \mathbf{r}_{j} / 2 a\right)-0.12$ with the constant giving rise to nonorthogonality of the eigenmodes. The linewidths of the superradiant and subradiant eigenmodes differ by two orders of magnitude, resulting in very different responses, and in both cases radically departing from the single-atom result. The substantially larger values of $1-g_{2}(0)$ compared to those of noninteracting atoms show enhanced antibunching due to interactions. In the subradiant case nonclassical effects are enhanced compared even with those of a single atom, with the nonclassical delay time of $g_{2}$ approximately 10 times larger than that of a single atom. Subradiant excitations can therefore provide much extended antibunching timescales compared with Rydbergatom based vapor cell devices [16], also avoiding twophoton excitations and the involvement of highly excited Rydberg states that are sensitive to electric and magnetic field gradients.

The SAP also provides an excellent description of the transient photon scattering rate $\langle\hat{n}(t)\rangle$ (insets to Fig. 1 and

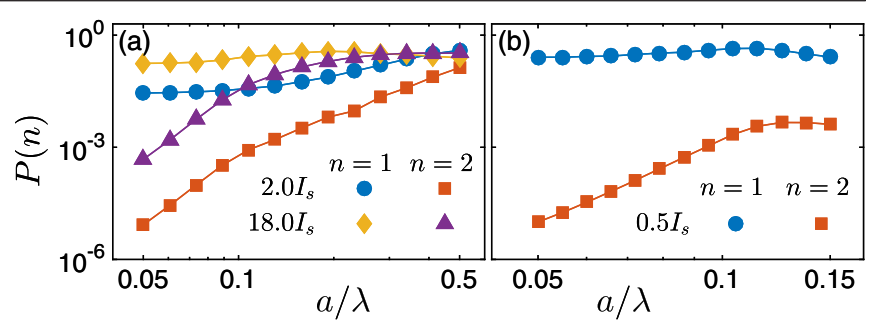

FIG. 2. Dipole blockade by the occupation weights $P(n)$ of states with $n=1$ and 2 excited atoms as a function of the lattice spacing $a$ in a $2 \times 3$ array, with (a) the superradiant, (b) subradiant LLI eigenmode targeted. For small $a, n=2$ states are suppressed by the blockade, regardless of intensity, but the blockade is weakened for larger $a$ and the occupation increases dramatically while the weight of $n=1$ states changes little in comparison. Drive intensity $N I_{\text {in }}$ given as a multiple of $I_{s}$.

Fig. S1 in the Supplemental Material [52]). The SAP for the photon scattering rate is $\langle\hat{n}(t)\rangle \approx n^{\left(v, \kappa^{\prime}\right)}(t)$, where $n^{(\gamma, \kappa)}(t) \equiv\left[I_{\text {in }} /\left(I_{\text {in }}+I_{s}\right)\right] g_{2}^{(\gamma, \kappa)}(t)$ is the photon scattering rate for a single, isolated atom [54,58].

The suppressed short-delay joint photon detection events in $g_{2}$ represent dipole blockade that inhibits multiple excitations of the collective atomic state, as illustrated in the excited-state atom-number distributions (Fig. 2). Already for a $2 \times 3$ array the multiple-excitation probability remains very low at small spacings. While the single-excitation weights are high, e.g., for the lattice spacing $a=0.05 \lambda$, the two-excitation weight is $\lesssim 10^{-5}$ at $N I_{\text {in }}=2 I_{s}$, but rapidly increases to 0.1 for $a=0.5 \lambda$, as the antibunching is reduced and the dipole blockade removed. The origin of the blockade can be understood also in the excitation spectrum $P(\Omega) \propto \int d \tau e^{i \Omega \tau} \sum_{j \ell} \gamma_{j \ell}\left\langle\hat{\sigma}_{j}^{+}(t+\tau) \hat{\sigma}_{\ell}^{-}(t)\right\rangle$ [inset to Fig. 3(b)] that shows how the second photon excitation is shifted due to the dipole-dipole interactions.

The accuracy and the regimes of validity of the SAP in both planar arrays and chains are analyzed in Fig. 3. The uniform phase profile of the drive across the atoms most strongly couples to the superradiant, uniform eigenmode, and we show the relative deviations $\eta \equiv \max _{\tau<\tau_{0}} \mid g_{2}(\tau) / b-$ $g_{2}^{\left(v, \kappa^{\prime}\right)}(\tau) \mid$ (calculated until $\tau_{0}$, such that for all $\tau \lesssim \tau_{0}$, $g_{2}(\tau)<1$; see also Fig. S2 in the Supplemental Material [52]). The SAP describes the behavior of $g_{2}(\tau)$ very well for $a \lesssim 0.1 \lambda$ and remains qualitatively accurate up to $a \sim$ $0.2 \lambda$ (a 9 atom chain gives similar results). The onset of the plateau around $a \approx 0.12 \lambda$, irrespective of light intensity, coincides with LLI eigenmode resonances overlapping with the superradiant mode. For $a \gtrsim 0.2 \lambda$, the SAP deviates from $g_{2}(\tau)$. The deviations as a function of $N$ in Fig. 3(a) show how the accuracy of the SAP decreases gradually in larger systems.

Increasing deviations for large values of $\tau$ for $a \gtrsim 0.2 \lambda$ are due to the presence of a persistent oscillation [a weak oscillation is also visible in Fig. 1(a)]. To understand this behavior, we look at the steady-state occupations of the LLI 

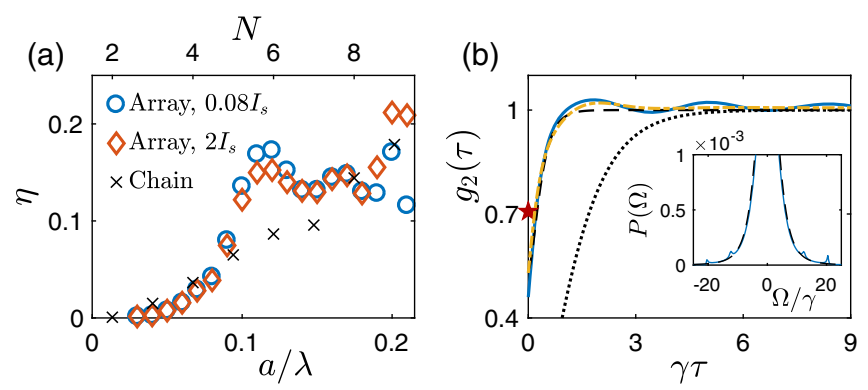

FIG. 3. Validity of the superatom picture and the effect of position fluctuations of the atoms for a field resonant with the uniform superradiant LLI mode. (a) Relative error $\eta$ of the SAP as a function of lattice spacing (bottom axis) for a $3 \times 3$ atom array at $N I_{\text {in }}=0.08 I_{s}$ (blue circles) and $N I_{\text {in }}=2 I_{s}$ (red diamonds), and as a function of atom number (top axis, crosses) for a chain at $a=0.15 \lambda, N I_{\text {in }}=0.08 I_{s}$; (b) position fluctuations of the atoms improve the accuracy of $\operatorname{SAP}(a=0.2 \lambda, 2 \times 3$ array): fixed atoms (blue solid line), fluctuating atoms with rms Gaussian density width $0.1 a$ at each lattice site (yellow dashed-dotted line), superatom (black dashed line), and single atom (black dotted line). The red star marks the noninteracting, interfering result of $g_{2}(0)$. Inset: Power spectrum for a $2 \times 3$ array $\left(I_{\text {in }}=2 I_{s}\right.$, $a=0.08 \lambda$ ) showing a superradiant central peak (SAP result: dashed line) with additional small excitations far off resonance.

modes for $\left\langle\sigma_{j}^{-}\right\rangle$, defined as [59] $L_{m} \equiv \sum_{j}\left|u_{m}\left(\mathbf{r}_{j}\right)\left\langle\sigma_{j}^{-}\right\rangle\right|^{2} /$ $\sum_{j \ell}\left|u_{\ell}\left(\mathbf{r}_{j}\right)\left\langle\sigma_{j}^{-}\right\rangle\right|^{2}$. The presence of the persistent oscillation coincides with a simultaneous non-negligible occupation of two eigenmodes. One can then qualitatively understand the effect of the two-mode interference from the linear combination

$g_{2}(\tau) \approx 1+b\left[C g_{2}^{\left(v_{1}, \kappa^{\prime}\right)}(\tau)+(1-C) g_{2}^{\left(v_{2}, \kappa_{2}\right)}(\tau)-1\right]$,

where the increasing contribution from the less radiant mode with increasing lattice spacing leads to deviations from the simple SAP at large $\tau$. Although we consider only chains and arrays, systems with higher symmetry such as rings [72] or configurations that optimize interactions offer the potential to more effectively target individual superatom resonances and enhance the photon blockade.

For atoms in optical lattices, proposals exist to produce a tight atom confinement [73], but generally the atomic positions fluctuate. We can take into account the position fluctuations in the numerics by ensemble-averaging over many stochastic realizations of randomly sampled atom positions in each lattice site [69]. We find in Fig. 3(b) that the accuracy of the superatom picture increases due to the fluctuations, as the oscillations resulting from the second eigenmode contribution are washed out. However, increasing position fluctuations eventually also start increasing $g_{2}(0)$.

The normalization of the SAP two-time correlation function at zero delay $g_{2}(0)$ in Eq. (5) represents the strength of nonclassical and correlated light emission of the

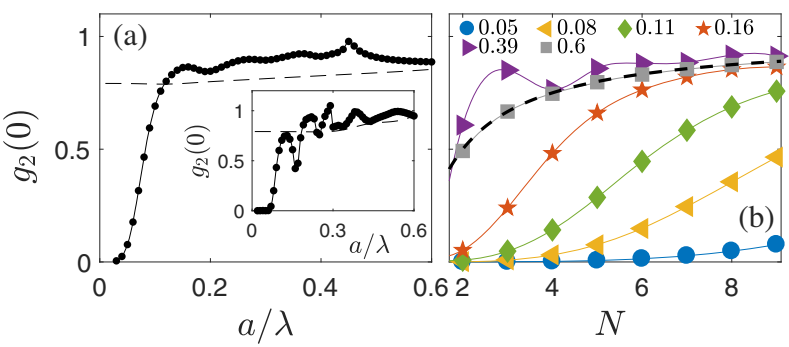

FIG. 4. Enhanced antibunching due to quantum correlations of light-induced dipole-dipole interactions in a 9-atom chain and $3 \times 3$ array. (a) $g_{2}(0)$ for a chain as a function of lattice spacing (inset: array) compared with noninteracting atoms (dashed line); (b) $g_{2}(0)$ as a function of atom number for chains with different lattice spacing $a / \lambda$ compared with noninteracting, noninterfering atoms (dashed line). Solid lines are guides for the eye.

atoms. For noninteracting atoms in the absence of multiple scattering, interference effects only slightly modify the result $g_{2}(0)=1-N^{-1}$. Strong light-mediated correlations, however, can substantially shift the value of $g_{2}(0)$, directly reflected in the antibunching of the emitted photons. In Fig. 4(a) we show $g_{2}(0)$ as a function of lattice spacing and atom number, with the drive tuned to the uniform LLI eigenmode. We find that light-mediated interactions enhance the nonclassical nature of light for small lattice spacing (up to $a \lesssim 0.15 \lambda$ ) that coincides with the regime where the SAP shows good accuracy over all values of $\tau$. For chains with large lattice spacing $(a \gtrsim 0.5 \lambda)$, lightmediated interactions between atoms are no longer sufficient to establish collective correlation effects, and $g_{2}(0)$ follows the noninteracting, noninterfering scaling $g_{2}(0)=$ $1-N^{-1}$ [Fig. 4(b)], with small or absent antibunching. In denser arrays, however, we find that nonclassical collective effects persist also as the atom number increases. For example, $g_{2}(0) \approx 0.08$ for a 9-atom chain with $a=0.05 \lambda$.

In Rydberg atoms, dipole blockade inhibits multiple excitations within the blockade radius $R$ [74]. Because of the long-range interactions present in our system, $R$ is in general not well defined. However, power-law-fit estimates of the dependence of $g_{2}(0)$ on the system size can be obtained from Fig. 4(b), resulting in $R$ of the order of $\lambda$, with a small roughly linear increase in $R$ with decreasing lattice spacing [75]. Correlations can be suppressed with a sufficiently broad laser [76] with increasing contributions from multiple modes [Eq. (6)] when the bandwidth notably exceeds $\gamma$.

The time-honored two-time correlation function (1) for joint photon emission events from a single atom reveals nonclassical resonance fluorescence of light [1,54]. Here we showed that the same functional form also describes emission from strongly coupled arrays of atoms, representing a superatom picture of correlated many-atom resonance fluorescence. For a single atom the suppression of joint photon emission events is a direct consequence of the 
fermionic statistics with $\left(\hat{\sigma}^{ \pm}\right)^{2}=0$ for the single excitation; after the photon emission the electron is in the ground state and cannot reemit before being excited again. For a manyatom system, the antibunching with $g_{2}(0) \simeq 0$ similarly represents the presence of only one excitation, where multiple excitations are inhibited by dipole blockadereminiscent of the fermionic character of multiple photon excitations of atoms in waveguides [37].

In the superatom picture of many-atom resonance fluorescence the strength of the correlations can surprisingly be determined by the underlying LLI collective excitation eigenmodes, even when the atoms are strongly driven by the incident laser. Such an effective collective description is quite different from representing the classical optical response of an atomic ensemble as a superatom in the limit of LLI by coupled collective eigenmodes [59,77]. Our analysis of the $g_{2}(\tau)$ correlations illustrates how relatively simple and intuitive representations could possibly more generally be extended to understand strongly correlated many-body phenomena in quantum optics far beyond linearly responding coupled classical dipoles.

Data used in this publication is available at [78].

We acknowledge financial support from Engineering and Physical Sciences Research Council (Grants No. EP/ S002952/1 and No. EP/P026133/1) and discussions with L. F. dos Santos.

Note added.-We have become aware of a related parallel theoretical work on the calculation of dipolar blockade in atom chains in Ref. [79].

[1] H. J. Carmichael and D. F. Walls, Proposal for the measurement of the resonant Stark effect by photon correlation techniques, J. Phys. B 9, L43 (1976).

[2] H. J. Kimble, M. Dagenais, and L. Mandel, Photon Antibunching in Resonance Fluorescence, Phys. Rev. Lett. 39, 691 (1977).

[3] H. J. Kimble, M. Dagenais, and L. Mandel, Multiatom and transit-time effects on photon-correlation measurements in resonance fluorescence, Phys. Rev. A 18, 201 (1978).

[4] M. Dagenais and L. Mandel, Investigation of two-time correlations in photon emissions from a single atom, Phys. Rev. A 18, 2217 (1978).

[5] D.F. Walls, Evidence for the quantum nature of light, Nature (London) 280, 451 (1979).

[6] X. T. Zou and L. Mandel, Photon-antibunching and subpoissonian photon statistics, Phys. Rev. A 41, 475 (1990).

[7] H. Paul, Photon antibunching, Rev. Mod. Phys. 54, 1061 (1982).

[8] E. Jakeman, E. R. Pike, P. N. Pusey, and J. M. Vaughan, The effect of atomic number fluctuations on photon antibunching in resonance fluorescence, J. Phys. A 10, L257 (1977).

[9] H. J. Carmichael, P. Drummond, P. Meystre, and D. F. Walls, Intensity correlations in resonance fluorescence with atomic number fluctuations, J. Phys. A 11, L121 (1978).
[10] D. Jaksch, J. I. Cirac, P. Zoller, S. L. Rolston, R. Côté, and M. D. Lukin, Fast Quantum Gates for Neutral Atoms, Phys. Rev. Lett. 85, 2208 (2000).

[11] M. D. Lukin, M. Fleischhauer, R. Cote, L. M. Duan, D. Jaksch, J. I. Cirac, and P. Zoller, Dipole Blockade and Quantum Information Processing in Mesoscopic Atomic Ensembles, Phys. Rev. Lett. 87, 037901 (2001).

[12] E. Urban, T. A. Johnson, T. Henage, L. Isenhower, D. D. Yavuz, T. G. Walker, and M. Saffman, Observation of Rydberg blockade between two atoms, Nat. Phys. 5, 110 (2009).

[13] A. Gaëtan, Y. Miroshnychenko, T. Wilk, A. Chotia, M. Viteau, D. Comparat, P. Pillet, A. Browaeys, and P. Grangier, Observation of collective excitation of two individual atoms in the Rydberg blockade regime, Nat. Phys. 5, 115 (2009).

[14] M. Saffman, T. G. Walker, and K. Mølmer, Quantum information with Rydberg atoms, Rev. Mod. Phys. 82, 2313 (2010).

[15] P. Schauß, M. Cheneau, M. Endres, T. Fukuhara, S. Hild, A. Omran, T. Pohl, C. Gross, S. Kuhr, and I. Bloch, Observation of spatially ordered structures in a two-dimensional Rydberg gas, Nature (London) 491, 87 (2012).

[16] F. Ripka, H. Kübler, R. Löw, and T. Pfau, A roomtemperature single-photon source based on strongly interacting Rydberg atoms, Science 362, 446 (2018).

[17] J. Javanainen, J. Ruostekoski, Y. Li, and S.-M. Yoo, Shifts of a Resonance Line in a Dense Atomic Sample, Phys. Rev. Lett. 112, 113603 (2014).

[18] S. E. Skipetrov and I. M. Sokolov, Absence of Anderson Localization of Light in a Random Ensemble of Point Scatterers, Phys. Rev. Lett. 112, 023905 (2014).

[19] W. Guerin, M. T. Rouabah, and R. Kaiser, Light interacting with atomic ensembles: Collective, cooperative and mesoscopic effects, J. Mod. Opt. 64, 895 (2017).

[20] J. Javanainen, J. Ruostekoski, Y. Li, and S.-M. Yoo, Exact electrodynamics versus standard optics for a slab of cold dense gas, Phys. Rev. A 96, 033835 (2017).

[21] O. Morice, Y. Castin, and J. Dalibard, Refractive index of a dilute Bose gas, Phys. Rev. A 51, 3896 (1995).

[22] J. Ruostekoski and J. Javanainen, Quantum field theory of cooperative atom response: Low light intensity, Phys. Rev. A 55, 513 (1997).

[23] S. Balik, A. L. Win, M. D. Havey, I. M. Sokolov, and D. V. Kupriyanov, Near-resonance light scattering from a highdensity ultracold atomic ${ }^{87} \mathrm{Rb}$ gas, Phys. Rev. A 87, 053817 (2013).

[24] J. Chabé, M.-T. Rouabah, L. Bellando, T. Bienaimé, N. Piovella, R. Bachelard, and R. Kaiser, Coherent and incoherent multiple scattering, Phys. Rev. A 89, 043833 (2014).

[25] J. Pellegrino, R. Bourgain, S. Jennewein, Y. R. P. Sortais, A. Browaeys, S. D. Jenkins, and J. Ruostekoski, Observation of Suppression of Light Scattering Induced by Dipole-Dipole Interactions in a Cold-Atom Ensemble, Phys. Rev. Lett. 113, 133602 (2014).

[26] C. C. Kwong, T. Yang, M. S. Pramod, K. Pandey, D. Delande, R. Pierrat, and D. Wilkowski, Cooperative Emission of a Coherent Superflash of Light, Phys. Rev. Lett. 113, 223601 (2014). 
[27] S. Jennewein, M. Besbes, N. J. Schilder, S. D. Jenkins, C. Sauvan, J. Ruostekoski, J.-J. Greffet, Y. R. P. Sortais, and A. Browaeys, Coherent Scattering of Near-Resonant Light by a Dense Microscopic Cold Atomic Cloud, Phys. Rev. Lett. 116, 233601 (2016).

[28] S. L. Bromley, B. Zhu, M. Bishof, X. Zhang, T. Bothwell, J. Schachenmayer, T. L. Nicholson, R. Kaiser, S. F. Yelin, M. D. Lukin, A. M. Rey, and J. Ye, Collective atomic scattering and motional effects in a dense coherent medium, Nat. Commun. 7, 11039 (2016).

[29] S. D. Jenkins, J. Ruostekoski, J. Javanainen, R. Bourgain, S. Jennewein, Y. R. P. Sortais, and A. Browaeys, Optical Resonance Shifts in the Fluorescence of Thermal and Cold Atomic Gases, Phys. Rev. Lett. 116, 183601 (2016).

[30] P. C. Bons, R. de Haas, D. de Jong, A. Groot, and P. van der Straten, Quantum Enhancement of the Index of Refraction in a Bose-Einstein Condensate, Phys. Rev. Lett. 116, 173602 (2016).

[31] W. Guerin, M. O. Araújo, and R. Kaiser, Subradiance in a Large Cloud of Cold Atoms, Phys. Rev. Lett. 116, 083601 (2016).

[32] S. Machluf, J. B. Naber, M. L. Soudijn, J. Ruostekoski, and R.J.C. Spreeuw, Collective suppression of optical hyperfine pumping in dense clouds of atoms in microtraps, Phys. Rev. A 100, 051801(R) (2019).

[33] L. Corman, J. L. Ville, R. Saint-Jalm, M. Aidelsburger, T. Bienaimé, S. Nascimbène, J. Dalibard, and J. Beugnon, Transmission of near-resonant light through a dense slab of cold atoms, Phys. Rev. A 96, 053629 (2017).

[34] R. J. Bettles, T. Ilieva, H. Busche, P. Huillery, S. W. Ball, N. L. R. Spong, and C.S. Adams, Collective mode interferences in light-matter interactions, arXiv:1808.08415.

[35] M. Hebenstreit, B. Kraus, L. Ostermann, and H. Ritsch, Subradiance Via Entanglement in Atoms with Several Independent Decay Channels, Phys. Rev. Lett. 118, 143602 (2017).

[36] R. Jones, R. Saint, and B. Olmos, Far-field resonance fluorescence from a dipole-interacting laser-driven cold atomic gas, J. Phys. B 50, 014004 (2017).

[37] Y.-X. Zhang and K. Mølmer, Theory of Subradiant States of a One-Dimensional Two-Level Atom Chain, Phys. Rev. Lett. 122, 203605 (2019).

[38] A. Grankin, P. O. Guimond, D. V. Vasilyev, B. Vermersch, and P. Zoller, Free-space photonic quantum link and chiral quantum optics, Phys. Rev. A 98, 043825 (2018).

[39] P.-O. Guimond, A. Grankin, D. V. Vasilyev, B. Vermersch, and P. Zoller, Subradiant Bell States in Distant Atomic Arrays, Phys. Rev. Lett. 122, 093601 (2019).

[40] R. J. Bettles, M. D. Lee, S. A. Gardiner, and J. Ruostekoski, Quantum and nonlinear effects in light transmitted through planar atomic arrays, arXiv:1907.07030.

[41] K. E. Ballantine and J. Ruostekoski, Subradiance-protected excitation spreading in the generation of collimated photon emission from an atomic array, Phys. Rev. Research 2, 023086 (2020).

[42] J. A. Needham, I. Lesanovsky, and B. Olmos, Subradianceprotected excitation transport, New J. Phys. 21, 073061 (2019).

[43] C. Qu and A.M. Rey, Spin squeezing and many-body dipolar dynamics in optical lattice clocks, Phys. Rev. A 100, 041602(R) (2019).
[44] L. A. Williamson and J. Ruostekoski, Optical response of atom chains beyond the limit of low light intensity: The validity of the linear classical oscillator model, Phys. Rev. Research 2, 023273 (2020).

[45] Y.-X. Zhang, C. Yu, and K. Mølmer, Subradiant bound dimer excited states of emitter chains coupled to a one dimensional waveguide, Phys. Rev. Research 2, 013173 (2020).

[46] L. Henriet, J. S. Douglas, D. E. Chang, and A. Albrecht, Critical open-system dynamics in a one-dimensional optical-lattice clock, Phys. Rev. A 99, 023802 (2019).

[47] A. Asenjo-Garcia, H. J. Kimble, and D. E. Chang, Optical waveguiding by atomic entanglement in multilevel atom arrays, Proc. Natl. Acad. Sci. U.S.A. 116, 25503 (2019).

[48] J. Rui, D. Wei, A. Rubio-Abadal, S. Hollerith, J. Zeiher, D. M. Stamper-Kurn, C. Gross, and I. Bloch, A subradiant optical mirror formed by a single structured atomic layer, Nature (London) 583, 369 (2020).

[49] A. Glicenstein, S. G. Ferioli, N. L. Brossard, I. FerrierBarbut, and A. Browaeys, Collective Shift in Resonant Light Scattering by a One-Dimensional Atomic Chain, Phys. Rev. Lett. 124, 253602 (2020).

[50] R. H. Lehmberg, Radiation from an $N$-atom system. I. General formalism, Phys. Rev. A 2, 883 (1970).

[51] G. S. Agarwal, Master-equation approach to spontaneous emission, Phys. Rev. A 2, 2038 (1970).

[52] See Supplemental Material at http://link.aps.org/ supplemental/10.1103/PhysRevLett.125.073602 for technical details, which includes Refs. [1,22,50,51,53-66].

[53] J. D. Jackson, Classical Electrodynamics, 3rd ed. (Wiley, New York, 1999).

[54] H. J. Carmichael and D. F. Walls, A quantum-mechanical master equation treatment of the dynamical Stark effect, J. Phys. B 9, 1199 (1976).

[55] J. Dalibard, Y. Castin, and K. Mølmer, Wave-Function Approach to Dissipative Processes in Quantum Optics, Phys. Rev. Lett. 68, 580 (1992).

[56] L. Tian and H. J. Carmichael, Quantum trajectory simulations of two-state behavior in an optical cavity containing one atom, Phys. Rev. A 46, R6801 (1992).

[57] R. Dum, P. Zoller, and H. Ritsch, Monte Carlo simulation of the atomic master equation for spontaneous emission, Phys. Rev. A 45, 4879 (1992).

[58] B. R. Mollow and M. M. Miller, The damped driven twolevel atom, Ann. Phys. (N.Y.) 52, 464 (1969).

[59] G. Facchinetti, S. D. Jenkins, and J. Ruostekoski, Storing Light with Subradiant Correlations in Arrays of Atoms, Phys. Rev. Lett. 117, 243601 (2016).

[60] M. R. Andrews, M.-O. Mewes, N. J. Van Druten, D. S. Durfee, D. M. Kurn, and W. Ketterle, Direct, nondestructive observation of a Bose condensate, Science 273, 84 (1996).

[61] H. J. Carmichael and K. Kim, A quantum trajectory unraveling of the superradiance master equation, Opt. Commun. 179, 417 (2000).

[62] H. J. Kimble and L. Mandel, Theory of resonance fluorescence, Phys. Rev. A 13, 2123 (1976).

[63] B. R. Mollow, Power spectrum of light scattered by twolevel systems, Phys. Rev. 188, 1969 (1969). 
[64] K. Mølmer, Y. Castin, and J. Dalibard, Monte Carlo wave-function method in quantum optics, J. Opt. Soc. Am. B 10, 524 (1993).

[65] J. P. Clemens, L. Horvath, B. C. Sanders, and H. J. Carmichael, Collective spontaneous emission from a line of atoms, Phys. Rev. A 68, 023809 (2003).

[66] J. Ruostekoski, M. J. Collett, R. Graham, and D. F. Walls, Macroscopic superpositions of Bose-Einstein condensates, Phys. Rev. A 57, 511 (1998).

[67] J. Javanainen, J. Ruostekoski, B. Vestergaard, and M. R. Francis, One-dimensional modeling of light propagation in dense and degenerate samples, Phys. Rev. A 59, 649 (1999).

[68] M. D. Lee, S. D. Jenkins, and J. Ruostekoski, Stochastic methods for light propagation and recurrent scattering in saturated and nonsaturated atomic ensembles, Phys. Rev. A 93, 063803 (2016).

[69] S. D. Jenkins and J. Ruostekoski, Controlled manipulation of light by cooperative response of atoms in an optical lattice, Phys. Rev. A 86, 031602(R) (2012).

[70] R. T. Sutherland and F. Robicheaux, Collective dipoledipole interactions in an atomic array, Phys. Rev. A 94, 013847 (2016).

[71] H. Carmichael, An Open Systems Approach to Quantum Optics, Lecture Notes in Physics Vol. 18 (Springer, Berlin, 1993).

[72] H. Dong, S.-W. Li, Z. Yi, G. S. Agarwal, and M. O. Scully, Photon-blockade induced photon anti-bunching in photosynthetic antennas with cyclic structures, arXiv:1608 .04364 .

[73] Y. Wang, S. Subhankar, P. Bienias, M. Łkacki, T.-C. Tsui, M. A. Baranov, A. V. Gorshkov, P. Zoller, J. V. Porto, and S. L. Rolston, Dark State Optical Lattice with a Subwavelength Spatial Structure, Phys. Rev. Lett. 120, 083601 (2018).

[74] D. Tong, S. M. Farooqi, J. Stanojevic, S. Krishnan, Y. P. Zhang, R. Côté, E. E. Eyler, and P. L. Gould, Local Blockade of Rydberg Excitation in an Ultracold Gas, Phys. Rev. Lett. 93, 063001 (2004).

[75] In Fig. 4(b) the $1 / r$ interaction is canceled due to $\hat{\mathbf{d}} \cdot \hat{\mathbf{x}}=1$, but different dipole orientations for which the $1 / r$ interaction is nonzero show analogous behavior.

[76] N. Takei, C. Sommer, C. Genes, G. Pupillo, H. Goto, K. Koyasu, H. Chiba, M. Weidemüller, and K. Ohmori, Direct observation of ultrafast many-body electron dynamics in an ultracold Rydberg gas, Nat. Commun. 7, 13449 (2016).

[77] G. Facchinetti and J. Ruostekoski, Interaction of light with planar lattices of atoms: Reflection, transmission, and cooperative magnetometry, Phys. Rev. A 97, 023833 (2018).

[78] L. A. Williamson, M. O. Borgh, and J. Ruostekoski, DOI: https://doi.org/10.17635/lancaster/researchdata/376

[79] A. Cidrim, T. S. do Espirito Santo, J. Schachenmayer, R. Kaiser, and R. Bachelard, preceding Letter, Photon Blockade with Ground-State Neutral Atoms, Phys. Rev. Lett. 125, 073601 (2020). 